\title{
Bone density, microarchitecture and stiffness in Caucasian and Caribbean Hispanic postmenopausal American women
}

\author{
Bin Zhou ${ }^{1}$, Ji Wang ${ }^{1}$, Emily M Stein ${ }^{2}$, Zhendong Zhang ${ }^{1,3}$, Kyle K Nishiyama ${ }^{2}$, Chiyuan A Zhang ${ }^{2}$, Thomas L Nickolas ${ }^{2}$, \\ Elizabeth Shane ${ }^{2}$ and X Edward Guo ${ }^{1}$
}

Hispanic Americans of Caribbean origin are a fast-growing subset of the US population, but there are no studies on bone density, microstructure and biomechanical integrity in this minority group. In this study, we aimed to compare Caucasian and Caribbean Hispanic postmenopausal American women with respect to these characteristics. Thirty-three Caribbean Hispanics were age-matched to thirty-three Caucasian postmenopausal women. At the lumbar spine, the Hispanic women had significantly lower areal bone mineral density (aBMD). At the radius by high-resolution peripheral quantitative computed tomography (HR-pQCT), there were minimal differences between Hispanic and Caucasian women. At the tibia, Hispanic women had lower trabecular volumetric bone density and trabecular number, and higher trabecular separation. Individual trabecula segmentation (ITS) analyses indicated that at the tibia, Hispanic women not only had significantly lower bone volume fraction, but also had significantly lower rod bone volume fraction, plate trabecular number, rod trabecular number and lower plate-plate, plate-rod and rod-rod junction densities compared to Caucasian women. The differences in bone quantity and quality contributed to lower whole bone stiffness at the radius, and both whole bone and trabecular bone stiffness at the tibia in Hispanic women. In conclusion, Hispanic women had poorer bone mechanical and microarchitectural properties than Caucasian women, especially at the load-bearing distal tibia.

Bone Research (2014) 2, 14016; doi:10.1038/boneres.2014.16; Published online: 16 September 2014

\section{INTRODUCTION}

Osteoporotic fractures are associated with significant morbidity and mortality in older men and women. ${ }^{1}$ It is estimated that the prevalence of osteoporosis will increase exponentially over the next several decades and that most of this increase will be in races other than Caucasian. $^{2}$ Bone density and risk of fracture varies according to race. $^{3-7}$ Fracture risk is lower among African American and Asian women compared with Caucasian and Hispanic women aged $50-80$ years, while there are no reported differences between Caucasian and Hispanic women. ${ }^{8}$ African Americans have higher areal bone mineral density (aBMD) as assessed by dual energy X-ray absorptiometry (DXA) than Caucasian women at the spine and hip. ${ }^{9}$ The associations between aBMD and risk of fracture may also differ among racial groups. For example, a one standard deviation decrease in aBMD was associated with a 1.49 times increase in fracture risk in Caucasian women, but a 1.37 times increase in African American women. ${ }^{3}$

Existing data on bone mass and fracture risk in Hispanic women are derived almost exclusively from those of Mexican descent. ${ }^{10-11}$ However, recent genetic ancestry studies have reported that the genome of Hispanics of Mexican descent is comprised of mostly European and Native American ancestry with very low African ancestry, while Caribbean Hispanics have significantly more African ancestry in their genome. ${ }^{12}$ Although higher African genetic ancestry could be associated with higher bone mass and lower fracture risk, there are virtually no data on Hispanic women from the Caribbean. Furthermore, bone geometry and structural properties also contribute to bone strength and therefore influence fracture risk ${ }^{13-17}$ and quantitative microstructural and mechanical data

${ }^{1}$ Bone Bioengineering Laboratory, Department of Biomedical Engineering, Columbia University, New York, USA; ${ }^{2}$ Division of Endocrinology, Department of Medicine, Columbia University, New York, USA and ${ }^{3}$ Department of Orthopedic Surgery, First Affiliated Hospital, School of Medicine Shihezi University, Shihezi, Xinjiang, China

Correspondence: XE Guo (ed.guo@columbia.edu); E Shane (es54@columbia.edu)

Received: 28 June 2014; Revised: 30 July 2014; Accepted: 30 July 2014 
in Hispanic American women, whether of Mexican or Caribbean descent, are currently not available. Although the incidence of hip fractures in Caucasians has declined in the past decade, ${ }^{18}$ it has increased ${ }^{19}$ or remained stable in Hispanic Americans. ${ }^{20}$ As the population and life expectancy of Hispanic Americans are expected to increase in the next few decades, ${ }^{21}$ there is a need to examine the characteristics of bone mass and bone microstructure in Hispanic Americans.

Recently, high-resolution peripheral quantitative computed tomography (HR-pQCT) has been used to evaluate three-dimensional (3D) microstructure at the distal radius and tibia. ${ }^{22-25}$ Analysis of $3 \mathrm{D}$ microstructural parameters furthers our understanding of bone quality beyond $\mathrm{ABMD}$ measured by DXA. HR-pQCT has previously been used to elucidate racial differences in bone structure. For example, postmenopausal Chinese-American women have thicker and denser cortical bone at the radius and higher trabecular bone volume fraction at the tibia than Caucasian women. ${ }^{26-27}$ In addition, HR-PQCT-based micro finite element ( $\mu \mathrm{FE})$ analyses have revealed that Chinese-American premenopausal women have higher estimated stiffness at the distal radius and tibia than Caucasian women. ${ }^{28}$ Individual trabecula segmentation (ITS) can be applied to HR-pQCT scans to segment the trabecular network into individual trabecular plates and rods, and provides an in-depth examination of the 3D microstructural characteristics through a series of independent ITS parameters. ${ }^{16}$ ITS analysis of HR-pQCT scans has shown that Chinese-American women have higher plate bone volume fraction and higher plate number density at the distal radius and tibia compared to Caucasian women, leading to a greater plate to rod ratio and greater trabecular connectivity. ${ }^{28-29}$

Because Caribbean Hispanic women have higher genetic admixture of African ancestry, we hypothesized that they would have better bone microstructure and bone strength than Caucasian women. We examined the differences in ABMD, bone microstructure and estimated bone mechanical competence in two groups of American postmenopausal women: Caucasian and Hispanic of Caribbean origin.

\section{MATERIALS AND METHODS}

\section{Subjects}

Postmenopausal women were recruited at Columbia University Medical Center (CUMC) by advertisements, flyers and at primary-care offices. Participants were excluded if they had a history of low trauma fractures, but there were no aBMD requirements for inclusion. Race/ethnicity was categorized by self-report. Inclusion criteria were self-reported full Caribbean Hispanic or Caucasian descent (all four grandparents) with residence in the United States. Women were excluded if they had a history of low trauma fracture, endocrinopathy (e.g., untreated hyperthyroidism, Cushing's syndrome, prolactinoma), celiac disease or other gastrointestinal diseases, abnormal mineral metabolism (e.g., osteomalacia, primary hyperparathyroidism), malignancy except for skin cancer and drug exposures that could affect bone metabolism (e.g., glucocorticoids, anticonvulsants, anticoagulants, methotrexate, aromatase inhibitors, thiazolidinediones). Women using hormone replacement therapy or raloxifene were permitted to participate. Women who had ever used teriparatide, or who had taken bisphosphonates for more than 1 year were excluded. All subjects provided written informed consent and the Institutional Review Board of CUMC approved this study.

\section{Areal bone mineral density by DXA}

We measured ABMD of the lumbar spine (LS), total hip (TH), femoral neck (FN), one-third radius (1/3R) and ultradistal radius (UDR) by DXA (QDR-4500; Hologic Inc., Walton, MA, at Columbia University Medical Center). Scans were performed by dedicated technologists, certified by the International Society for Clinical Densitometry. $T$ scores compared participants with young-normal Caucasian populations, provided by each manufacturer.

HR-pQCT images of the distal radius and distal tibia All participants were scanned at CUMC by HR-PQCT (XtremeCT; Scanco Medical, Brüttisellen, Switzerland) at the non-dominant forearm and matching ankle as previously described. ${ }^{24,30-32}$ The HR-pQCT measurement included 110 slices, corresponding to a $9.02 \mathrm{~mm}$ section along the axial direction, with a voxel size of $82 \mu \mathrm{m}$. After each scan, the operator examined the reconstructed images and participants with severe movement artifact were rescanned. Each image was assigned an image grade from 1 (no motion) to 5 (severe blurring and streaks) by a trained technician. Repeat scans were performed if the original scan was graded 4 or 5. Quality control was provided by scanning the European Forearm Phantom. The mineralized phase was thresholded according to the standard patient evaluation protocol. ${ }^{33}$ We measured total, cortical and trabecular areas, total, cortical, and trabecular volumetric BMD, cortical thickness, trabecular number, trabecular thickness and trabecular separation. ${ }^{34}$ The in vivo reproducibility of HR-PQCT in our center was $0.55 \%-1.25 \%$ for density measures, $0.16 \%$ $1.25 \%$ for area measures and $3.65 \%-5.22 \%$ for trabecular microstructure measures (unpublished data). The accuracy of HR-PQCT measures has been validated through comparisons with $\mu \mathrm{CT}$ measured parameters on cadaveric subjects. ${ }^{22}$ 
ITS-based morphological analyses of HR-pQCT images We used ITS on the trabecular compartment to evaluate the plate- and rod-related microstructural parameters (Figure 1). Briefly, digital topological analysis-based skeletonization ${ }^{35}$ was first applied to transform the trabecular bone image into a reduced structural skeleton while preserving the topology. ${ }^{36-38}$ Digital topological classification was performed and each skeletal voxel was uniquely classified as either a surface or a curve type. Using an iterative reconstruction method, each voxel of the original image was classified belonging to either a plate (surface) or a rod (curve). Based on the 3D evaluations of the trabecular bone network, the following parameters were evaluated: plate and rod bone volume fraction (pBV/TV and rBV/TV); plate and rod number density (pTb.N and rTb.N, $\mathrm{mm}^{-1}$ ); plate-rod ratio (PR ratio); axial bone volume fraction (aBV/TV); plate and rod trabecular thickness (pTb.Th and rTb.Th, $\mathrm{mm}$ ); plate trabecular surface (pTb.S, $\mathrm{mm}^{2}$ ); rod trabecular length (rTb. $\ell$, $\mathrm{mm})$; trabecular connection densities between plate-plate, plate-rod, and rod-rod (P-P Junc.D, P-R Junc.D and R-R Junc.D, $\mathrm{mm}^{-3}$ ). We also performed a direct measure of bone volume fraction (BV/TV) calculated as the voxels occupied by bone divided by total voxels. The predictive ability of ITS at limited resolutions was also validated through a comparison of ITS parameters based on HR-pQCT images and those based on $\mu \mathrm{CT}$ images for the same subject. ${ }^{39}$

Finite element analyses of HR-pQCT images

Each thresholded HR-PQCT whole bone image and trabecular bone compartment image of the distal radius and tibia was converted to a $\mu \mathrm{FE}$ model by converting each voxel to an eight-node brick element. Bone tissue was modeled as an isotropic, linear elastic material with a Young's modulus of 15 GPa and a Poisson's ratio of $0.3 .{ }^{40}$ For each model of whole bone or trabecular bone, a uniaxial compression test was performed to calculate the
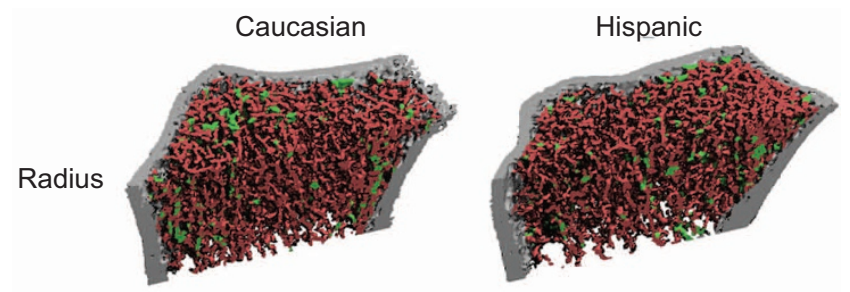

Tibia
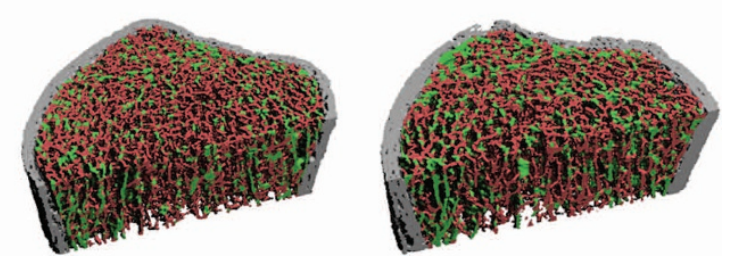

Figure 1. Representative images indicating ITS analyses of individual trabecular plates (green) and rods (red) in radius and tibia for Caucasian and Hispanic women. reaction force under a displacement equal to $1 \%$ of the bone segment height along the axial direction. Whole bone stiffness, defined as reaction force divided by the applied displacement, characterizes the mechanical competence of both cortical and trabecular compartments and is closely related to whole bone strength ${ }^{41}$ and fracture risk. ${ }^{42-44}$ Similarly, trabecular bone stiffness characterizes the mechanical competence of trabecular bone compartment. We also calculated the percent of the load carried by the cortical compartment at the distal and proximal surfaces of the bone segments. All the $\mu \mathrm{FE}$ analyses were performed by using a customized elementby-element, preconditioned conjugate gradient solver. ${ }^{45}$

\section{Statistical analysis}

Results for DXA, HR-PQCT, ITS and FE are expressed as mean \pm standard deviation (s.d.). To examine group differences according to race/ethnicity, $a B M D$, microstructure and FE parameters were first compared by analysis of variance. Analyses were then adjusted using a general linear model with $\mathrm{BMI}$ as covariate to assess its influence on the comparisons. We considered $P<0.05$ to be statistically significant. All analysis was performed using SAS (Version 9.1; SAS Institute Inc, Cary, NC, USA).

\section{RESULTS}

We studied 66 postmenopausal women: 33 Hispanic women of Caribbean origin and 33 age-matched ( \pm 2 years) Caucasian women who had participated in a previous study of bone quality. ${ }^{24,31-32}$ On average, Hispanic women were shorter $(P=0.002)$ and had a higher $\mathrm{BMI}$ than Caucasian women ( $P=0.03)$. Years since menopause, family history of osteoporosis, use of vitamin D supplements, serum 25OHD level, raloxifene, bisphosphonates and hormone-replacement therapy did not differ between Hispanic and Caucasian women. Calcium supplements, alcohol and tobacco use were significantly lower in Hispanic women (Table 1).

Measurements of aBMD by DXA differed between the groups, but only at the spine (Table 2). Compared with Caucasian women, Hispanic women had lower aBMD at the lumbar spine (by 7.8\%), with no differences at the forearm and hip. Their lumbar spine aBMD remained significantly lower after adjusting for BMI.

By HR-pQCT of the distal radius, bone size was similar and there were no differences in trabecular parameters between Caucasian and Hispanic women. Cortical density was lower in Hispanic women (by 4.2\%) (Table 3). After adjusting for BMl, the lower cortical density remained significant in Hispanic women, who also had lower cortical thickness and lower total bone density than Caucasian women. In contrast, at the tibia, cortical thickness and density did not differ between Caucasian and Hispanic women, 
Table 1. Characteristics of the study cohort

\begin{tabular}{|c|c|c|c|}
\hline Parameters & Caucasian ( $n=33)$ & Hispanic $(n=33)$ & $P$-value \\
\hline Age/years & $65.5 \pm 5.6$ & $64.9 \pm 6.2$ & 0.700 \\
\hline Height/cm & $161.9 \pm 5.7$ & $156.6 \pm 6.8$ & 0.002 \\
\hline Weight/kg & $69.3 \pm 13.5$ & $72.9 \pm 14.0$ & 0.300 \\
\hline $\mathrm{BMI} /\left(\mathrm{kg} \cdot \mathrm{m}^{-2}\right)$ & $26.6 \pm 5.6$ & $29.8 \pm 5.5$ & 0.030 \\
\hline Years since menopausal & $16.0 \pm 8.0$ & $16.0 \pm 8.0$ & 0.900 \\
\hline $\mathrm{PTH} /\left(\mathrm{pg} \cdot \mathrm{mL}^{-1}\right)$ & $42.2 \pm 18.9$ & $41.2 \pm 20.6$ & 0.800 \\
\hline Vitamin D supplements, total daily dose/IU & $685.0 \pm 1456.0$ & $393.0 \pm 557.0$ & 0.300 \\
\hline Calcium supplements, total daily dose/mg & $1355.0 \pm 751.0$ & $866.0 \pm 478.0$ & 0.003 \\
\hline Serum $250 \mathrm{HD} /\left(\mathrm{ng} \cdot \mathrm{mL}^{-1}\right)$ & $33.0 \pm 11.4$ & $26.9 \pm 11.7$ & 0.130 \\
\hline Alcohol use (beverages per day) & $1.0 \pm 1.6$ & $0.4 \pm 0.6$ & 0.047 \\
\hline Tobacco use (packs per year) & $164.0 \pm 288.0$ & $52.0 \pm 111.0$ & 0.040 \\
\hline Family history of osteoporosis/\% & 64.0 & 36.0 & 0.058 \\
\hline Raloxifene/\% & 3.0 & 0 & 0.940 \\
\hline \multicolumn{4}{|l|}{ Bisphosphonates/\% } \\
\hline Past & 6.0 & 3.0 & 1.000 \\
\hline Current & 0 & 6.0 & 0.700 \\
\hline \multicolumn{4}{|l|}{ Hormone replacement therapy (HRT)/\% } \\
\hline Past & 36.0 & 30.0 & 1.000 \\
\hline Current & 0 & 6.0 & 0.400 \\
\hline
\end{tabular}

before or after adjustment for BMI. However, Hispanic women had lower trabecular density (by 12.2\%, $P=0.04$ ), lower trabecular number (by $9.4 \%, P=0.03$ ) and greater trabecular separation (by $16.2 \%, P=0.02$ ) than Caucasian women, and these differences persisted after adjusting for BMI. After adjusting for BMl, total bone density was also lower in Hispanic than Caucasian women $(P=0.04)$.

Trabecular microstructure was evaluated further by ITS (Table 4). At the radius, the pattern mirrored that seen by the standard HR-pQCT analysis: there were no betweengroups differences in trabecular plate or rod microstructure, before or after adjusting for BMI. However, at the tibia, Hispanic women had significantly lower directly measured bone volume fraction, rod BV/TV, plate and rod trabecular number, and lower trabecular plate and rod connectivity including P-P, P-R and R-R junction densities, but significantly higher rod trabecular length ( $r T b . \ell$ ) than Caucasian women (Figure 1). After adjusting for BMI, all differences remained significant.

The microstructural differences revealed by HR-pQCT and ITS were consistent with differences in mechanical properties between the racial groups (Table 5). At the radius, there was no difference in unadjusted calculated mechanical parameters between Caucasian and Hispanic women. After adjusting for BMI, whole bone stiffness of Hispanic women tended to be lower than Caucasian women. At the tibia, trabecular bone stiffness was significantly lower in Hispanic women, before and after adjusting for BMI. After adjusting for BMI, whole bone stiffness was significantly lower in Hispanic women. In addition, compared to Caucasian women, a significantly higher proportion of the mechanical load in Hispanic women was carried by cortical bone at the distal ends, both before and after adjusting for BMI.

\section{DISCUSSION}

The expanding population of Hispanic Americans in the United States combined with data demonstrating the potential for an increasing number of future fractures in this population underscores the importance of examining bone density and quality in this group. ${ }^{8}$ To our knowledge, most studies comparing skeletal differences among Caucasian and Hispanic women have focused on aBMD by DXA and primarily evaluated Hispanics of Mexican descent. ${ }^{46-47}$ In this study, we utilized standard in vivo HRPQCT and advanced imaging technique ITS in addition to DXA, and compared microarchitectural and mechanical properties between Caucasian postmenopausal women and Hispanic postmenopausal women of Caribbean descent. With HR-pQCT, Putman et $a l .{ }^{48}$ found

Table 2. Comparison of DXA measurements between Caucasian and Hispanic women. Data was presented as mean \pm s.d., before and after adjusting for BMI

\begin{tabular}{|c|c|c|c|c|}
\hline DXA aBMD & Caucasian $(n=33)$ & Hispanic $(n=33)$ & $P$-value & $P$-value (BMI adjusted) \\
\hline Lumbar spine $/\left(\mathrm{g} \cdot \mathrm{cm}^{-2}\right)$ & $0.959 \pm 0.127$ & $0.884 \pm 0.157$ & 0.04 & 0.005 \\
\hline Femoral neck $/\left(\mathrm{g} \cdot \mathrm{cm}^{-2}\right)$ & $0.703 \pm 0.069$ & $0.727 \pm 0.133$ & 0.35 & 0.800 \\
\hline Total hip/(g.cm $\left.{ }^{-2}\right)$ & $0.835 \pm 0.104$ & $0.862 \pm 0.141$ & 0.38 & 0.580 \\
\hline $1 / 3$ radius $/\left(\mathrm{g} \cdot \mathrm{cm}^{-2}\right)$ & $0.626 \pm 0.064$ & $0.613 \pm 0.086$ & 0.48 & 0.200 \\
\hline Ultradistal radius $/\left(\mathrm{g} \cdot \mathrm{cm}^{-2}\right)$ & $0.389 \pm 0.067$ & $0.394 \pm 0.071$ & 0.79 & 0.280 \\
\hline
\end{tabular}


Table 3. Comparison of the morphological parameters as shown by HR-pQCT analysis between Caucasian and Hispanic women. Data was presented as mean \pm s.d., before and after adjusting for BMI

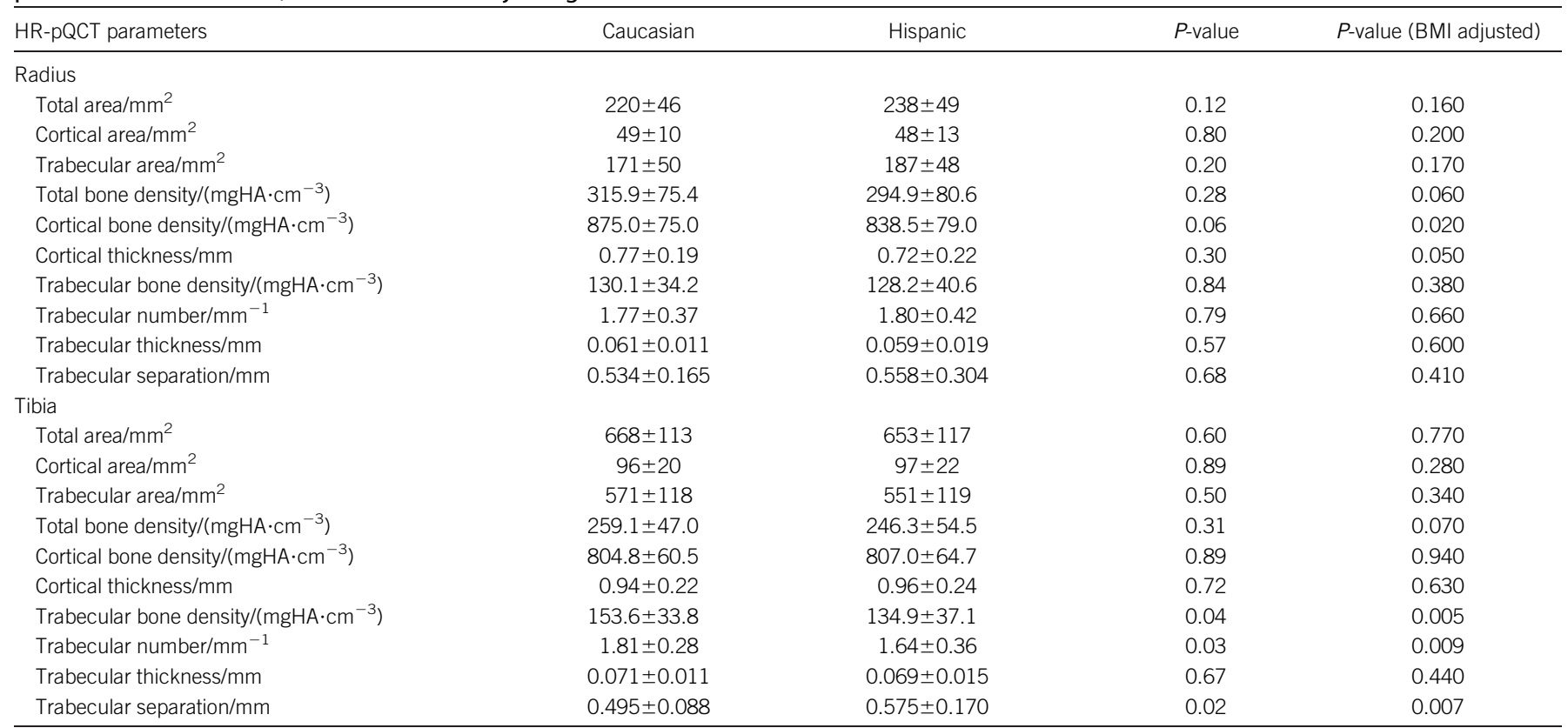

that African American postmenopausal women had larger bone size, higher trabecular number and higher cortical thickness than Caucasian women, differences that could result in stronger bone and lower fracture risk in African American women. As Caribbean Hispanics have significant African ancestry in their genome, ${ }^{12}$ we

Table 4. Microstructural measurements as evaluated by ITS between Caucasian and Hispanic women. Data was presented as mean $\pm s . d .$, before and after adjusting for BMI

\begin{tabular}{|c|c|c|c|c|}
\hline ITS parameters & Caucasian & Hispanic & $P$-value & $P$-value (BMI adjusted) \\
\hline \multicolumn{5}{|l|}{ Radius } \\
\hline $\mathrm{BV} / \mathrm{TV}$ & $0.228 \pm 0.049$ & $0.230 \pm 0.060$ & 0.900 & 0.530 \\
\hline $\mathrm{pBV} / \mathrm{TV}$ & $0.065 \pm 0.032$ & $0.066 \pm 0.033$ & 0.910 & 0.600 \\
\hline $\mathrm{rBV} / \mathrm{TV}$ & $0.163 \pm 0.030$ & $0.164 \pm 0.038$ & 0.920 & 0.650 \\
\hline PR ratio & $0.400 \pm 0.210$ & $0.400 \pm 0.190$ & 0.960 & 0.660 \\
\hline $\mathrm{aBV} / \mathrm{TV}$ & $0.079 \pm 0.025$ & $0.075 \pm 0.026$ & 0.450 & 0.180 \\
\hline pTb.N/mm $m^{-1}$ & $1.290 \pm 0.180$ & $1.270 \pm 0.220$ & 0.700 & 0.320 \\
\hline $\mathrm{rTb} . \mathrm{N} / \mathrm{mm}^{-1}$ & $1.840 \pm 0.150$ & $1.820 \pm 0.200$ & 0.640 & 0.320 \\
\hline pTb.Th/mm & $0.203 \pm 0.009$ & $0.209 \pm 0.015$ & 0.070 & 0.150 \\
\hline rTb.Th/mm & $0.213 \pm 0.007$ & $0.213 \pm 0.009$ & 0.980 & 0.980 \\
\hline $\mathrm{pTb} . \mathrm{S} / \mathrm{mm}^{2}$ & $0.137 \pm 0.013$ & $0.142 \pm 0.034$ & 0.400 & 0.400 \\
\hline $\mathrm{rTb} . \ell / \mathrm{mm}$ & $0.680 \pm 0.029$ & $0.708 \pm 0.128$ & 0.230 & 0.140 \\
\hline R-R Junc. D/mm ${ }^{-3}$ & $2.950 \pm 0.750$ & $2.910 \pm 0.790$ & 0.830 & 0.480 \\
\hline P-R Junc. D/mm ${ }^{-3}$ & $2.890 \pm 1.030$ & $2.900 \pm 1.150$ & 0.980 & 0.530 \\
\hline P-P Junc. $D / \mathrm{mm}^{-3}$ & $1.320 \pm 0.550$ & $1.310 \pm 0.580$ & 0.990 & 0.490 \\
\hline \multicolumn{5}{|l|}{ Tibia } \\
\hline $\mathrm{BV} / \mathrm{TV}$ & $0.269 \pm 0.042$ & $0.243 \pm 0.048$ & 0.030 & 0.003 \\
\hline $\mathrm{pBV} / \mathrm{TV}$ & $0.117 \pm 0.038$ & $0.111 \pm 0.041$ & 0.580 & 0.220 \\
\hline $\mathrm{rBV} / \mathrm{TV}$ & $0.152 \pm 0.030$ & $0.133 \pm 0.035$ & 0.020 & 0.003 \\
\hline PR ratio & $0.820 \pm 0.360$ & $0.950 \pm 0.560$ & 0.270 & 0.440 \\
\hline aBV/TV & $0.116 \pm 0.027$ & $0.109 \pm 0.030$ & 0.320 & 0.150 \\
\hline pTb.N/mm $m^{-1}$ & $1.510 \pm 0.130$ & $1.450 \pm 0.150$ & 0.090 & 0.020 \\
\hline $\mathrm{rTb} . \mathrm{N} / \mathrm{mm}^{-1}$ & $1.790 \pm 0.150$ & $1.690 \pm 0.210$ & 0.030 & 0.020 \\
\hline pTb.Th/mm & $0.218 \pm 0.011$ & $0.220 \pm 0.013$ & 0.440 & 0.990 \\
\hline rTb.Th/mm & $0.217 \pm 0.007$ & $0.216 \pm 0.008$ & 0.550 & 0.760 \\
\hline pTb.S $/ \mathrm{mm}^{2}$ & $0.151 \pm 0.018$ & $0.160 \pm 0.027$ & 0.100 & 0.130 \\
\hline $\mathrm{rTb} . \ell / \mathrm{mm}$ & $0.654 \pm 0.023$ & $0.678 \pm 0.031$ & 0.001 & 0.000 \\
\hline R-R Junc. $D / \mathrm{mm}^{-3}$ & $2.570 \pm 0.790$ & $2.160 \pm 0.840$ & 0.040 & 0.040 \\
\hline P-R Junc. D/mm ${ }^{-3}$ & $3.700 \pm 0.870$ & $3.180 \pm 0.950$ & 0.020 & 0.004 \\
\hline P-P Junc. D/mm ${ }^{-3}$ & $2.000 \pm 0.510$ & $1.760 \pm 0.540$ & 0.060 & 0.010 \\
\hline
\end{tabular}


Table 5. Comparison of the mechanical properties as evaluated by FE between Caucasian and Hispanic women. Data was presented as mean \pm s.d., before and after adjusting for BMI

\begin{tabular}{|c|c|c|c|c|}
\hline FEA parameters & Caucasian & Hispanic & $P$-value & $P$-value (BMI adjusted) \\
\hline \multicolumn{5}{|l|}{ Radius } \\
\hline Whole bone stiffness $/\left(\mathrm{N} \cdot \mathrm{mm}^{-1}\right)$ & $71381 \pm 16631$ & $68097 \pm 21669$ & 0.500 & 0.080 \\
\hline Trabecular bone stiffness $/\left(\mathrm{N} \cdot \mathrm{mm}^{-1}\right)$ & $9194 \pm 7339$ & $9606 \pm 7636$ & 0.830 & 0.720 \\
\hline Cortical load_distal/\% & $0.430 \pm 0.080$ & $0.450 \pm 0.120$ & 0.290 & 0.500 \\
\hline Cortical load_proximal/\% & $0.920 \pm 0.070$ & $0.930 \pm 0.070$ & 0.910 & 0.440 \\
\hline \multicolumn{5}{|l|}{ Tibia } \\
\hline Whole bone stiffness $/\left(\mathrm{N} \cdot \mathrm{mm}^{-1}\right)$ & $218696 \pm 43938$ & $204608 \pm 46070$ & 0.210 & 0.009 \\
\hline Trabecular bone stiffness $/\left(\mathrm{N} \cdot \mathrm{mm}^{-1}\right)$ & $87508 \pm 36280$ & $68897 \pm 30296$ & 0.030 & 0.004 \\
\hline Cortical load—distal/\% & $0.270 \pm 0.070$ & $0.350 \pm 0.100$ & 0.000 & 0.005 \\
\hline Cortical load_proximal/\% & $0.700 \pm 0.100$ & $0.740 \pm 0.080$ & 0.090 & 0.070 \\
\hline
\end{tabular}

hypothesized that our Hispanic subjects would have better bone microarchitecture and stiffness than Caucasian women. Contrary to our hypothesis, we found that Caribbean Hispanic postmenopausal women had lower areal BMD by DXA at the spine, both before and after adjustment for BMI, and that $\mathrm{ABMD}$ did not differ at the hip or forearm. By HR-pQCT, they had lower total and cortical volumetric density and a trend toward thinner cortices, but no differences in trabecular volumetric BMD or microarchitecture at the radius. In contrast, at the tibia, a different pattern emerged-cortical parameters were similar, while trabecular volumetric density was lower and trabecular microarchitecture was of poorer quality. ITS, which decomposes the trabecular network into individual trabecular plates and rods and directly calculates plate/rod related structural parameters such as plate/rod number and thickness, plate surface and rod length, provided additional insights into trabecular morphology at the tibia, beyond those detected by HR-pQCT. Specifically, ITS revealed lower directly measured trabecular bone volume fraction, fewer plates and rods and lower plate and rod connectivity in Hispanic than Caucasian women. These trabecular differences translated into lower estimated whole bone and trabecular bone stiffness at the tibia in Hispanic than Caucasian women. The ITS findings advanced the standard morphological analysis of HR-PQCT and are consistent with previous clinical results with ITS technology. ${ }^{28,49-50}$

Although contrary to our hypothesis, the aBMD differences between Caucasian and Caribbean Hispanic women in this study are similar to previous reports of Hispanic women of Mexican descent. After adjusting for BMI, Morton et al. ${ }^{46}$ reported that Hispanic women had significantly lower aBMD at the lumbar spine but not at the hip. In contrast to our findings, studies from the National Health and Examination Survey reported that Hispanic women of Mexican descent had lower aBMD at the hip compared to Caucasian women. ${ }^{51} \mathrm{BMI}$ was found to be significantly correlated with aBMD. ${ }^{52}$ Putman et al. ${ }^{53}$ also found the differences in radius cortical thickness and tibia bone size between patient with cystic fibrosis and controls were from BMI. In this study, to eliminate the BMI effect and examine the underlying bone microstructural and mechanical differences between Hispanic and Caucasian women, we incorporated BMl as covariant in the general linear model.

At the loading bearing distal tibia, the Caucasian women in our study, despite being lighter, had better trabecular bone quality than Hispanic women in terms of plate and rod trabecular number, P-P, P-R and R-R junction densities. We found that the differences in $B V / T V$ at the tibia were due to lower rod BV/TV. Interestingly, previous studies have shown that rBV/TV does not contribute significantly to overall trabecular bone stiffness. ${ }^{54}$ However, microstructure evaluated by both HR-PQCT and ITS at the radius, a site which does not experience significant loading, did not differ significantly between Caucasian and Hispanic women, excepting that axial BV/TV tended to be higher in Caucasian women. The effect of the clinical covariates known to affect bone quality was also examined in the general linear model, including calcium supplements, tobacco and alcohol use. The differences between Hispanic and Caucasian women remained the same after adjusting those parameters.

To investigate potential differences in bone mechanical competence between the groups, we performed FE analyses on both the whole bone and the trabecular compartment. FE analysis of the whole bone was used to examine overall bone mechanical competence, which is related to bone size, geometry and microstructure. After adjusting for BMl, whole bone stiffness at the radius was significantly higher in Caucasian than Hispanic women, while trabecular bone stiffness was similar. Thus, the higher radius bone stiffness in Caucasians is likely related to their thicker cortices. At the tibia, Caucasian women had both higher trabecular and whole bone stiffness than Hispanic women, likely due to better trabecular microstructure.

This study has several limitations. The number of participants was small and recruitment was not populationbased. Thus, the differences we observed could be due to ascertainment bias, as women concerned about their 
bone health might have been more likely to participate. We studied only postmenopausal women, and thus we cannot address whether the differences we observed reflect peak differences in bone mass and microarchitecture or arise during and after transition through menopause. DNA was not collected from the participants, so we cannot assess whether genetic contributions to bone mass, microarchitecture and stiffness differ between Caucasian and Hispanic women. In addition, we did not assess factors such as physical activity which may affect bone microstructural properties and should be considered in future studies. The racial identification is made through self-reporting, which is not necessarily ideal. A recent study has shown that the self-reporting race has an overall $87.2 \%$ accuracy in classifying all races and $72 \%$ accuracy particular in Hispanics. ${ }^{55}$ Our future studies should consider using genotyping a set of ancestry informative markers for racial characterization. Factors such as body composition including fat and lean mass were also not considered in this current study. They could add interesting additional information and will be considered in our further studies. From a technical perspective, the voxel size of the HR-pQCT images, $82 \mu \mathrm{m}$, is close to the width of a single trabecula, and several routine HR-pQCT trabecular parameters are calculated, rather than directly measured.

In addition, there are several strengths of this study. To our knowledge, it is the only study to evaluate volumetric bone mass, microarchitecture and stiffness in Hispanic women from the Caribbean basin. Hispanic women were matched for age to Caucasian women. It is also the only study to apply both standard HR-pQCT and advanced ITS measurements and FEA to this group of women. All three techniques have been systemically validated against the gold standard of $\mu \mathrm{CT}$ imaging. $22,39,56$ The finding that Hispanic women have similar, or in some respects worse, skeletal integrity than Caucasian women is surprising as they were significantly heavier and their genetic background includes African ancestry. It is also of concern and suggests that they may be at similar or even higher risk for postmenopausal osteoporotic fractures. Thus, the results of this study suggest that larger, population-based studies are needed to confirm these findings and to examine the contributions of genetics, obesity, cultural and environmental factors to their skeletal deficits.

In summary, we used HR-PQCT, ITS and $\mu \mathrm{FE}$ analysis to compare bone mass, microstructure and stiffness in Caucasian and Caribbean Hispanic postmenopausal women. Hispanic women had lower areal BMD by DXA at the spine, and poorer bone mechanical and microarchitecture properties than Caucasian women, especially at the load-bearing distal tibia. These results suggest that bone quality is poorer than expected and raises the concern that risk of osteoporotic fractures may be higher than expected in Hispanic postmenopausal women of Caribbean origin, who comprise a significant proportion of the Hispanic population in the United States.

\section{Competing interests}

The authors declare no conflict of interest.

\section{Acknowledgements}

This work was supported by NIH Grants R01 AR051376 (XEG), NIH R01 AR058004 (XEG, ES), NIH U01 AR055968 (ES) and by the Thomas L. Kempner and Katheryn C. Patterson Foundation.

\section{References}

1 Nam HS, Kweon SS, Choi JS et al. Racial/ethnic differences in bone mineral density among older women. J Bone Miner Metab 2013; 31: 190-198.

2 Burge R, Dawson-Hughes B, Solomon DH, Wong JB, King A, Tosteson A. Incidence and economic burden of osteoporosis-related fractures in the United States, 2005-2025. J Bone Miner Res 2007; 22: 465-475.

3 Cauley JA, Lui LY, Ensrud KE et al. Bone mineral density and the risk of incident nonspinal fractures in black and white women. JAMA 2005; 293 : 2102-2108

4 Cauley JA, Wu L, Wampler NS et al. Clinical risk factors for fractures in multi-ethnic women: the Women's Health Initiative. J Bone Miner Res 2007; 22: 1816-1826.

5 Cummings SR, Cauley JA, Palermo L et al. Racial differences in hip axis lengths might explain racial differences in rates of hip fracture. Osteoporos Int 1994; 4: 226-229.

6 Mackey DC, Eby JG, Harris F et al. Prediction of clinical non-spine fractures in older black and white men and women with volumetric BMD of the spine and areal BMD of the hip: the Health, Aging, and Body Composition Study. J Bone Miner Res 2007; 22: 1862-1868.

7 Nelson DA, Barondess DA, Hendrix SL, Beck TJ. Cross-sectional geometry, bone strength, and bone mass in the proximal femur in black and white postmenopausal women. J Bone Miner Res 2000; 15: 1992-1997.

8 Barrett-Connor E, Siris ES, Wehren LE et al. Osteoporosis and fracture risk in women of different ethnic groups. J Bone Miner Res 2005; 20: 185-194.

9 Hochberg MC. Racial differences in bone strength. Trans Am Clin Climatol Assoc 2007; 118: 305-315

10 Looker AC, Melton LJ 3rd, Harris TB, Borrud LG, Shepherd JA. Prevalence and trends in low femur bone density among older US adults: NHANES 2005-2006 compared with NHANES III. J Bone Miner Res 2010; 25: 64-71.

11 Nelson DA, Beck TJ, Wu G et al. Ethnic differences in femur geometry in the women's health initiative observational study. Osteoporos Int 2011; 22: 1377-1388

12 Peralta CA, Li YM, Wassel C et al. Differences in albuminuria between Hispanics and Whites: an evaluation by genetic ancestry and country of origin the multi-ethnic study of atherosclerosis. Circ Cardiovasc Genet 2010; 3: 240-247.

13 Fields AJ, Eswaran SK, Jekir MG, Keaveny TM. Role of trabecular microarchitecture in whole-vertebral body biomechanical behavior. $J$ Bone Miner Res 2009; 24: 1523-1530.

14 Fields AJ, Lee GL, Liu XS, Jekir MG, Guo XE, Keaveny TM. Influence of vertical trabeculae on the compressive strength of the human vertebra. $J$ Bone Miner Res 2011; 26: 263-269.

15 Liu XS, Cohen A, Shane E et al. Individual trabeculae segmentation (ITS)based morphological analysis of high-resolution peripheral quantitative computed tomography images detects abnormal trabecular plate and rod 
microarchitecture in premenopausal women with idiopathic osteoporosis. J Bone Miner Res 2010; 25: 1496-1505.

16 Liu XS, Sajda P, Saha PK et al. Complete volumetric decomposition of individual trabecular plates and rods and its morphological correlations with anisotropic elastic moduli in human trabecular bone. J Bone Miner Res 2008; 23: 223-235.

17 Liu XS, Sajda P, Saha PK, Wehrli FW, Guo XE. Quantification of the roles of trabecular microarchitecture and trabecular type in determining the elastic modulus of human trabecular bone. J Bone Miner Res 2006; 21: 1608-1617.

18 Brauer CA, Coca-Perraillon M, Cutler DM, Rosen AB. Incidence and mortality of hip fractures in the United States. JAMA 2009; 302: 1573-1579.

19 Zingmond DS, Melton LJ 3rd, Silverman SL. Increasing hip fracture incidence in California Hispanics, 1983 to 2000. Osteoporos Int 2004; 15: 603-610.

20 Wright NC, Saag KG, Curtis JR et al. Recent trends in hip fracture rates by race/ethnicity among older US adults. J Bone Miner Res 2012; 27: 2325-2332.

21 Cauley JA. Defining ethnic and racial differences in osteoporosis and fragility fractures. Clin Orthop Relat Res 2011; 469: 1891-1899.

22 Liu XS, Zhang XH, Sekhon KK et al. High-resolution peripheral quantitative computed tomography can assess microstructural and mechanical properties of human distal tibial bone. J Bone Miner Res 2010; 25: 746-756.

23 Nishiyama KK, Macdonald HM, Buie HR, Hanley DA, Boyd SK. Postmenopausal women with osteopenia have higher cortical porosity and thinner cortices at the distal radius and tibia than women with normal aBMD: an in vivo HR-pQCT study. J Bone Miner Res 2010; 25: 882-890.

24 Stein EM, Liu XS, Nickolas TL et al. Abnormal microarchitecture and reduced stiffness at the radius and tibia in postmenopausal women with fractures. J Bone Miner Res 2010; 25: 2572-2581.

$25 \mathrm{Vu}$ TD, Wang XF, Wang Q et al. New insights into the effects of primary hyperparathyroidism on the cortical and trabecular compartments of bone. Bone 2013; 55: 57-63.

26 Walker MD, Liu XS, Stein E et al. Differences in bone microarchitecture between postmenopausal Chinese-American and white women. J Bone Miner Res 2011; 26: 1392-1398.

27 Walker MD, Saeed I, McMahon DJ et al. Volumetric bone mineral density at the spine and hip in Chinese American and White women. Osteoporos Int 2012; 23: 2499-2506.

28 Liu XS, Walker MD, McMahon DJ et al. Better skeletal microstructure confers greater mechanical advantages in Chinese-American women versus white women. J Bone Miner Res 2011; 26: 1783-1792.

29 Cong E, Walker MD. The Chinese skeleton: insights into microstructure that help to explain the epidemiology of fracture. Bone Research 2014; 2 : 14009.

30 Liu XS, Cohen A, Shane E et al. Bone density, geometry, microstructure, and stiffness: relationships between peripheral and central skeletal sites assessed by DXA, HR-pQCT, and cQCT in premenopausal women. J Bone Miner Res 2010; 25: 2229-2238.

31 Stein EM, Liu XS, Nickolas TL et al. Microarchitectural abnormalities are more severe in postmenopausal women with vertebral compared to nonvertebral fractures. J Clin Endocrinol Metab 2012; 97: E1918-E1926.

32 Stein EM, Liu XS, Nickolas TL et al. Abnormal microarchitecture and stiffness in postmenopausal women with ankle fractures. J Clin Endocrinol Metab 96: 2041-2048.

33 Boutroy S, Bouxsein ML, Munoz F, Delmas PD. In vivo assessment of trabecular bone microarchitecture by high-resolution peripheral quantitative computed tomography. J Clin Endocrinol Metab 2005; 90: 6508-6515.

34 Laib A, Hauselmann HJ, Ruegsegger P. In vivo high resolution 3D-QCT of the human forearm. Technol Health Care 1998; 6: 329-337.
35 Saha PK, Chaudhuri BB, Dutta D, Majumder DD. A new shape preserving parallel thinning algorithm for 3D digital images. Pattern Recog 1997; 30: 1939-1955.

36 Saha PK, Chaudhuri BB, Chanda B, Majumder DD. Topology preservation in 3D digital space. Patt Recog 1994; 27: 295-300.

37 Saha PK, Chaudhuri BB. 3D digital topology under binary transformation with applications. Comput Vis Image Understand 1996; 63: 418-429.

38 Saha PK, Chaudhuri BB. Detection of 3-D simple points for topology preserving transformations with application to thinning. IEEE Trans Pattern Anal Mach Intell 1994; 16: 1028-1032.

39 Liu XS, Shane E, McMahon DJ, Guo XE. Individual trabecula segmentation (ITS)-based morphological analysis of microscale images of human tibial trabecular bone at limited spatial resolution. J Bone Miner Res 2011; 26: 2184-2193.

40 Guo XE, Goldstein SA. Is trabecular bone tissue different from cortical bone tissue? Forma 1997; 12: 185-196.

41 Macneil JA, Boyd SK. Bone strength at the distal radius can be estimated from high-resolution peripheral quantitative computed tomography and the finite element method. Bone 2008; 42: 1203-1213.

42 Stein EM, Liu XS, Nickolas TL et al. Abnormal microarchitecture and reduced stiffness at the radius and tibia in postmenopausal women with fractures. J Bone Miner Res 2010; 25: 2572-2581.

43 Melton LJ 3rd, Christen D, Riggs BL et al. Assessing forearm fracture risk in postmenopausal women. Osteoporos Int 2010; 21: 1161-1169.

44 Vilayphiou N, Boutroy S, Sornay-Rendu E et al. Finite element analysis performed on radius and tibia HR-pQCT images and fragility fractures at all sites in postmenopausal women. Bone 2010; 46: 1030-1037.

45 Hollister SJ, Brennan JM, Kikuchi N. A homogenization sampling procedure for calculating trabecular bone effective stiffness and tissue level stress. J Biomech 1994; 27: 433-444.

46 Morton DJ, Barrett-Connor E, Kritz-Silverstein D, Wingard DL, Schneider DL. Bone mineral density in postmenopausal Caucasian, Filipina, and Hispanic women. Int J Epidemiol 2003; 32: 150-156.

47 Taaffe DR, Villa ML, Holloway L, Marcus R. Bone mineral density in older non-Hispanic Caucasian and Mexican-American women: relationship to lean and fat mass. Ann Hum Biol 2000; 27: 331-344.

48 Putman MS, Yu EW, Lee H et al. Differences in skeletal microarchitecture and strength in African-American and white women. J Bone Miner Res 2013; 28: 2177-2185.

49 Liu XS, Cohen A, Shane E et al. Individual trabeculae segmentation (ITS)based morphological analyses of high resolution peripheral quantitative computed tomography images detect abnormal trabecular plate and rod microarchitecture in premenopausal women with idiopathic osteoporosis. J Bone Miner Res 2010; 25: 1496-1505.

50 Walker MD, Liu XS, Zhou B et al. Premenopausal and postmenopausal differences in bone microstructure and mechanical competence in ChineseAmerican and white women. J Bone Miner Res 2013; 28: 1308-1318.

51 Looker AC, Orwoll ES, Johnston CC Jr et al. Prevalence of low femoral bone density in older U.S. adults from NHANES III. J Bone Miner Res 1997; 12: 1761-1768.

52 Mohamed EI, Tarantino U, Promenzio L, de Lorenzo A. Predicting bone mineral density of postmenopausal healthy and cirrhotic Italian women using age and body mass index. Acta Diabetol 2003; 40(Suppl 1): S23-S28.

53 Putman MS, Milliren CE, Derrico N et al. Compromised bone microarchitecture and estimated bone strength in young adults with cystic fibrosis. J Clin Endocrinol Metab 2014; 99: 3399-3407.

54 Zhou B, Liu XS, Wang J, Lu XL, Fields AJ, Guo XE. Dependence of mechanical properties of trabecular bone on plate-rod microstructure determined by individual trabecula segmentation (ITS). J Biomech 2014; 47: 702-708. 
55 Hall JB, Dumitrescu L, Dilks HH, Crawford DC, Bush WS. Accuracy of administratively-assigned ancestry for diverse populations in an electronic medical record-linked biobank. PLoS One 2014; 9: e99161.

56 MacNeil JA, Boyd SK. Accuracy of high-resolution peripheral quantitative computed tomography for measurement of bone quality. Med Eng Phys 2007; 29: 1096-1105.
(1) $(\Theta)$ This work is licensed under a Creative Commons Attribution(c) ${ }_{\mathrm{BY}} \mathrm{NC}$ ND NonCommercial-NoDerivs 3.0 Unported License. The images or other third party material in this article are included in the article's Creative Commons license, unless indicated otherwise in the credit line; if the material is not included under the Creative Commons license, users will need to obtain permission from the license holder to reproduce the material. To view a copy of this license, visit http://creativecommons.org/licenses/by-nc-nd/3.0/ 